\title{
A contemporary comparison of the effect of shunt type in hypoplastic left heart syndrome on the hemodynamics and outcome at Fontan completion
}

\author{
Jean A. Ballweg, MD, ${ }^{\mathrm{a}}$ Troy E. Dominguez, MD, ${ }^{\mathrm{a}}$ Chitra Ravishankar, MD, ${ }^{\mathrm{a}} \mathrm{J}$. William Gaynor, MD, ${ }^{\mathrm{a}}$ \\ Susan C. Nicolson, MD, ${ }^{a}$ Thomas L. Spray, MD, ${ }^{a}$ and Sarah Tabbutt, MD, $\mathrm{PhD}^{\mathrm{b}}$
}

\begin{abstract}
Objective: We previously reported no difference in morbidity or mortality in a cohort of infants undergoing stage 1 and 2 reconstructions for hypoplastic left heart syndrome with either a modified Blalock-Taussig shunt or a right ventricular to pulmonary artery conduit. This article compares the hemodynamics and perioperative course at the time of the Fontan completion and reports longer-term survival for this cohort.
\end{abstract}

\begin{abstract}
Methods: We retrospectively reviewed the hospital records of all patients who underwent stage 1 reconstruction between January 2002 and May 2005 and subsequent surgical procedures, as well as cross-sectional analysis of hospital survivors.

Results: A total of 176 patients with hypoplastic left heart syndrome or a variant underwent stage 1 reconstruction with either modified Blalock-Taussig shunt $(n=114)$ or right ventricular to pulmonary artery conduit $(n=62)$. Shunt selection was at the discretion of the surgeon. The median duration of follow-up was 58 months (range 1-87 months). By Kaplan-Meier analysis, shunt type did not influence survival or freedom from transplant at 5 years (right ventricular to pulmonary artery conduit $61 \%$; $95 \%$ confidence limit, $47-72$ vs modified Blalock-Taussig shunt $70 \% ; 95 \%$ confidence limit, $60-77 ; P=.55)$. A total of 107 patients underwent Fontan (69 modified Blalock-Taussig shunts and 38 right ventricular to pulmonary artery conduits) with $98 \%(105 / 107)$ early survival. Patients with a right ventricular to pulmonary artery conduit shunt pre-Fontan had higher pulmonary artery (13 $\pm 8 \mathrm{~mm} \mathrm{Hg}$ vs $11 \pm 3 \mathrm{~mm} \mathrm{Hg}, P=.026)$ and common atrial $(8 \pm 2.3 \mathrm{~mm} \mathrm{Hg}$ vs $6.8 \pm 2.7 \mathrm{~mm} \mathrm{Hg}$, $P=.039)$ pressures. By echocardiography evaluation, there was more qualitative moderate to severe ventricular dysfunction (right ventricular to pulmonary artery conduit 31\% [12/36] vs modified Blalock-Taussig shunt 17\% [11/67], $P=.05$ ) and moderate to severe atrioventricular valve regurgitation (right ventricular to pulmonary artery conduit $40 \%$ [14/35] vs modified Blalock-Taussig shunt $16 \%$ [11/67], $P=.01$ ) in the right ventricular to pulmonary artery conduit group. Use of diuretic therapy, angiotensin-converting enzyme inhibition, reflux medications, and tube feedings were not different between groups. Overall, 5 patients underwent heart transplantation (right ventricular to pulmonary artery conduit 4 vs modified Blalock-Taussig shunt $1, P=.1$ ) before Fontan. There was no difference in age or weight at Fontan, bypass time, intensive care unit or hospital length of stay, postoperative pleural effusions, or need for reoperation between groups.
\end{abstract}

Conclusions: Interim analyses continue to suggest there is no survival advantage of one shunt type compared with the other. Longer-term follow-up of a randomized patient population remains of utmost importance. (J Thorac Cardiovasc Surg 2010;140:537-44)

In the absence of transplantation, hypoplastic left heart syndrome (HLHS) and variants are palliated with a staged approach. In stage 1 reconstruction (S1R), pulmonary blood

\footnotetext{
From the Children's Hospital of Philadelphia, ${ }^{\text {a }}$ Philadelphia, Pa; and University of California San Francisco, ${ }^{\mathrm{b}}$ San Francisco, Calif.

Disclosures: None.

Read at the Eighty-ninth Annual Meeting of the American Association for Thoracic Surgery, May 11, 2009, Boston, Massachusetts.

Received for publication May 10, 2009; revisions received Jan 11, 2010; accepted for publication March 21, 2010.

Address for reprints: Jean A Ballweg, MD, University of Tennessee Health Science Center, Le Bonheur Children's Hospital, Heart Institute, 50 N Dunlap St, Memphis, TN 38103 (E-mail: jballweg@uthsc.edu). $0022-5223 / \$ 36.00$

Copyright (c) 2010 Published by Elsevier Inc. on behalf of The American Association for Thoracic Surgery

doi:10.1016/j.jtcvs.2010.03.045
}

flow can be supplied via a modified Blalock-Taussig shunt (mBTS) or a right ventricle to pulmonary artery (RV-PA) conduit. A contemporary comparison of the immediate hemodynamics after S1R with both shunt types in a nonrandomized study did not demonstrate benefit of one shunt type versus the other. ${ }^{1}$ Longer-term follow-up studies through stage 2 reconstruction (S2R) also have not demonstrated advantage of one shunt type versus the other. ${ }^{2,3}$

To date, there are few studies that define the morbidity and mortality at Fontan using both shunt types. A study using historical controls ${ }^{4}$ and a single study with contemporary controls $^{5}$ have not demonstrated significant advantage to one shunt type at the time of Fontan completion. We therefore undertook the current study to better define the outcomes at the time of Fontan completion and longer-term 

Abbreviations and Acronyms
AVVR $=$ atrioventricular valve regurgitation
HLHS = hypoplastic left heart syndrome
mBTS $=$ modified Blalock-Taussig shunt
$\mathrm{RV}-\mathrm{PA}=$ right ventricle to pulmonary artery
$\mathrm{S} 1 \mathrm{R}=$ stage 1 reconstruction
$\mathrm{S} 2 \mathrm{R}=$ stage 2 reconstruction

mortality with a contemporary cohort of both shunt types. Our study objectives include the difference between the 2 shunt groups with regard to (1) preoperative hemodynamics and patient characteristics, (2) Fontan morbidity and mortality, and (3) interim mortality of the entire study cohort.

\section{MATERIALS AND METHODS}

This study was a cross-sectional case series including all patients who underwent an S1R for HLHS or variants at The Children's Hospital of Philadelphia between January 1, 2002, and May 1, 2005 ( $\mathrm{n}=176)$, with particular attention paid to S2R hospital survivors $(n=119)$. This was a consent-waived study approved by The Children's Hospital of Philadelphia Institutional Review Board. Sources of information included the hospital medical records, cardiac center and cardiac intensive care databases, records and reports provided by referring cardiologists, and pertinent information obtained from the parents on interview. Patient data were collected through April 16, 2009.

All patients were born with HLHS or variants with a single ventricle and obstruction to systemic blood flow. Anatomic diagnoses were divided into (1) usual HLHS with mitral stenosis or atresia; (2) malaligned atrioventricular canal defect with a single right ventricle; (3) ventricular septal defect or double-outlet right ventricle with mitral stenosis or atresia; (4) single left ventricle with systemic outflow obstruction, such as double-inlet left ventricle with transposition of the great arteries; and (5) other, including truncus arteriosus with single ventricle.

We reviewed the most recent clinic visit or admission note for use of digoxin, diuretic therapy, angiotensin-converting enzyme inhibitor agents, and anti-reflux medications. We recorded data on need for supplemental feeding via nasogastric or gastrostomy tubes and need for occupational, speech, or physical therapy. Reported developmental delay was recorded.

We reviewed the most recent echocardiographic report before Fontan completion for the study patients. Echocardiographers were not blinded as to the type of initial shunt type chosen for pulmonary blood. Reported qualitative assessments of systolic ventricular function, atrioventricular valve regurgitation (AVVR), and neoaortic valve regurgitation were recorded. Systolic ventricular function was documented as normal, mild, moderate, or severely depressed. AVVR and neoaortic valve regurgitation were documented as none, mild, moderate, or severe in nature.

Oximetry and hemodynamic data obtained at cardiac catheterization with conscious sedation or general anesthesia before Fontan completion were collected. Measured data included hemoglobin concentration, venous and arterial saturations, common atrial pressure, pulmonary artery pressure, and single ventricle end-diastolic pressure. If magnetic resonance imaging was used in place of or in addition to cardiac catheterization before Fontan completion, this was recorded.

The operative techniques for S1R and S2R have been described. ${ }^{1,2}$ As previously described, initial shunt type was at the discretion of the surgeon and included the use of the mBTS for all patients with single left ventricle and a preference for the RV-PA conduit in patients with aortic atresia. ${ }^{1}$ At the time of S2R, no patient was left with antegrade flow. Type of
Fontan (lateral tunnel or extracardiac) procedure was at the discretion of the surgeon. Age and size at Fontan and comparative operative data, including total support time, aortic crossclamp time, and deep hypothermic circulatory arrest time, were recorded. Postoperative data, including length of intubation, incidence of reintubation, and cardiac intensive care unit and hospital lengths of stay, were also recorded. Need for extracorporeal membrane oxygenation, reoperation, or cardiac catheterization before discharge or listing for transplantation was documented. Other perioperative morbid events, including placement of chest tubes and wound infections, were documented. The incidence of rehospitalization was obtained to assess the postdischarge morbidity. For rehospitalization, the indications for admission, length of stay, invasive procedures performed, and outcome were ascertained from the medical records or the parents directly.

Data are presented as medians (ranges) or means ( \pm standard deviation) for continuous variables and as a count and percentage for dichotomous variables. Continuous variables were compared by using the Mann-Whitney test and Student $t$ test. Fisher's exact test and chi-square analyses were used for dichotomous and categoric variables. The Kaplan-Meier method was used to generate survival curves beginning at birth using a composite end point of death or transplantation that occurred up to the time of final data collection. Comparison between survival curves by shunt type was performed using the log-rank method. Multivariate modeling using Cox proportional hazards regression was used to adjust for differences in baseline characteristics between shunt types to determine the association between shunt type and the composite outcome. Variables were included in the model for adjustment if the significant difference between shunt types was $P$ less than .1. All data were analyzed by using STATA 9.2 software (STATA Corp, College Station, Tex).

\section{RESULTS \\ Patient Population}

The study population consisted of 176 patients who underwent S1R for HLHS or variants with a single ventricle and systemic outflow obstruction. Anatomic subtype has been reported. ${ }^{1}$ Cardiac anatomy and previously reported risk factors stratified by shunt type can be found in Table 1. In addition, since the introduction of the RV-PA conduit in early 2002, there has been consistent use of both shunts throughout the study period (Figure 1). Fluctuations over time may reflect the patient population (specific anatomy), surgeon, or recent successes or failures of a particular shunt type. The RV-PA conduit was preferred for patients with aortic atresia or an aberrant right subclavian artery, and the mBTS was preferred for patients with a single LV. Patients were relatively evenly distributed between shunt types with respect to previously reported risk factors (exclusive of cardiac anatomy) with the exception of noncardiac anomalies (Table 1).

Follow-up data are complete through April 16, 2009. Incomplete data are available for 6 patients in the RV-PA conduit group who underwent Fontan completion at other institutions. No patient has been lost to follow-up. An mBTS was placed in 114 patients, and an RV-PA conduit was placed in 62 patients. ${ }^{1}$ The outcomes through S2R have been described. ${ }^{2}$ At the time of S2R analysis, 78 of 80 patients palliated with mBTS had undergone S2R, with 2 patients awaiting further palliation to Glenn physiology. Similarly, 46 of 47 patients palliated with RV-PA conduit 
TABLE 1. Baseline cardiac anatomy and previously reported risk factors stratified by shunt type before stage 1 reconstruction

\begin{tabular}{|c|c|c|c|}
\hline & $\operatorname{mBTS}(n=114)$ & RV-PA $(n=62)$ & $\boldsymbol{P}$ \\
\hline \multicolumn{4}{|l|}{ Cardiac anatomy } \\
\hline HLHS & $66(57.9 \%)$ & $55(88.7 \%)$ & $<.001$ \\
\hline Unbalanced AVC & $11(9.7 \%)$ & $4(6.5 \%)$ & .58 \\
\hline VSD with MS/MA & $18(15.8 \%)$ & $2(3.2 \%)$ & .012 \\
\hline Single LV & $17(14.9 \%)$ & $0(0 \%)$ & .001 \\
\hline Other & $2(1.8 \%)$ & $1(1.6 \%)$ & 1 \\
\hline Aortic atresia & $35(30.5 \%)$ & $43(69.4 \%)$ & $<.001$ \\
\hline \multicolumn{4}{|l|}{$\begin{array}{l}\text { Previously reported } \\
\text { risk factors }\end{array}$} \\
\hline Birth weight $<2.5 \mathrm{~kg}$ & $22(19.3 \%)$ & $14(22.6 \%)$ & .7 \\
\hline Noncardiac anomalies & $7(2.6 \%)$ & $7(11.3 \%)$ & .035 \\
\hline Genetic syndrome & $5(4.4 \%)$ & $3(4.8 \%)$ & 1 \\
\hline $\begin{array}{l}\text { Associated cardiac } \\
\text { risk factors }\end{array}$ & $15(13.5 \%)$ & $3(4.9 \%)$ & .08 \\
\hline Preoperative shock & $9(8 \%)$ & $5(8 \%)$ & 1 \\
\hline \multicolumn{4}{|c|}{$\begin{array}{l}A V C \text {, Atrioventricular canal; } V S D \text {, ventricular septal defect; } M S \text {, mitral stenosis; } M A \text {, } \\
\text { mitral atresia. Shunt type }=\text { RV-PA compared with mBTS baseline. } \\
\text { Other = unbalance AVC with truncus arteriosus and interrupted aortic arch, superior } \\
\text { ventricles with AVC and transposition of the great arteries }\{\mathrm{S}, \mathrm{D}, \mathrm{L}\} \text { with mitral steno- } \\
\text { sis. Additional cardiac risk factors were considered to be an intact atrial septum requir- } \\
\text { ing emergency intervention, moderate to severe AVVR, and severe ventricular } \\
\text { dysfunction at birth. Noncardiac anomalies included scimitar syndrome, gastroschisis, } \\
\text { duodenal atresia, and tracheoesophageal fistula. }\end{array}$} \\
\hline
\end{tabular}

had undergone S2R with 1 patient awaiting further palliation. Of the 116 S2R survivors, 107 have undergone Fontan completion (mBTS 69/72; RV-PA 38/40; $P=.79$ ) (Figure 2). Four other procedures have been performed: One patient in the mBTS cohort underwent conversion to 2-ventricle palliative repair, and 2 patients in the RV-PA conduit cohort underwent transplantation. Five patients are awaiting Fontan completion: 3 in the mBTS group and 2 in the RV-PA conduit group. Two of the 3 patients in the mBTS cohort remain palliated with shunt physiology. Interstage mortality, defined as death after hospital discharge from S2R and before Fontan completion, was not significantly different between shunt types (mBTS 6.3\% [5/80]; RV-PA $6.4 \%$ [3/47]; $P=.7$ ).

\section{Preoperative Morbidity}

We compared age, patient size, medication use, and need for supplemental feeding at the time of Fontan completion. There was no difference in the age of patients between shunt types (mBTS 2.7 years [range, 1.5-4.4 years]; RV-PA 2.9 years [range, 1.9-3.9 years], $P=.4$ ). In addition, patient size as measured by weight and body surface area at the time of surgery was not different between shunt types (Table 2). The use of diuretic therapy, angiotensinconverting enzyme inhibitors, reflux medications, and tube feedings was not different between groups (Table 2). The use of digoxin was significantly more frequent in patients in the RV-PA group (mBTS 234\% [17/72], RV-PA 43\% [16/37]; $P=.04)$. A total of 32 patients were receiving occupational/physical or speech therapy at the time of Fontan completion. There was no difference in shunt type and need for therapy or diagnosis of developmental delay (mBTS 28\% [20/72], RV-PA 40\% [15/38]; $P=.13$ ).

\section{Preoperative Echocardiographic Data}

The RV-PA cohort had significantly more moderate to severe qualitative ventricular systolic dysfunction in the RVPA cohort (mBTS 16\% [11/67]; RV-PA 31\% [11/36], $P=.05)$. There was also more moderate to severe AVVR in the RV-PA cohort (mBTS 16\% [11/67]; RV-PA 40\%

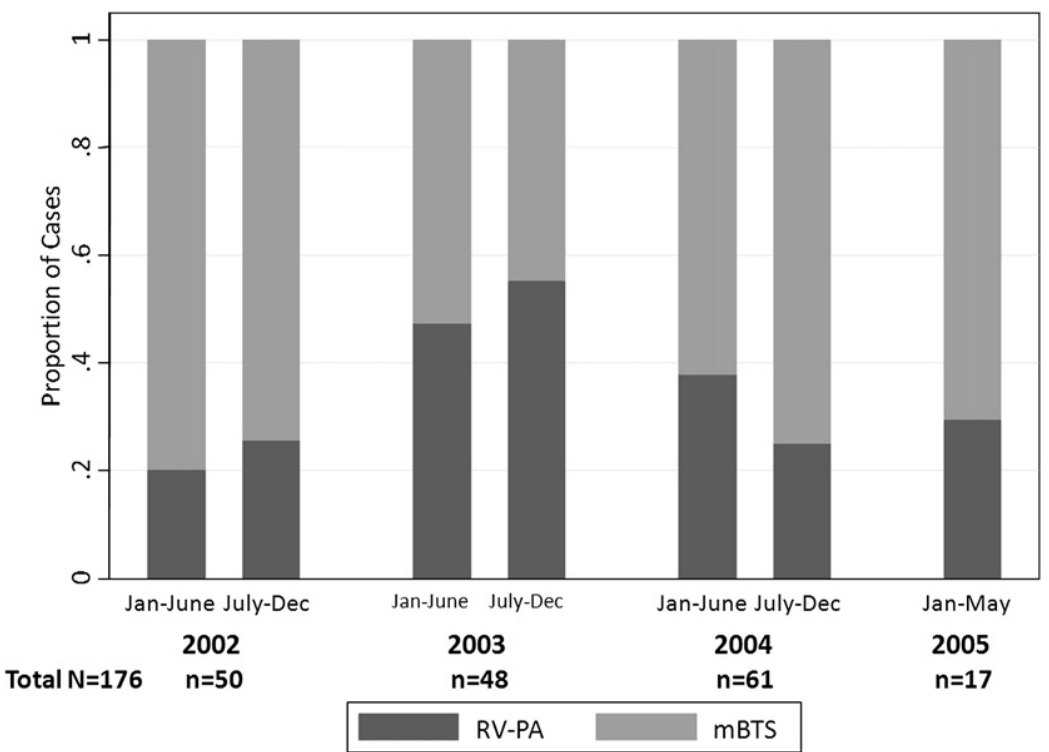

FIGURE 1. Distribution of shunt types over time ( $\mathrm{n}=$ total stage I reconstruction during the time block). All time blocks are 6 months, except the last is 5 months. mBTS (light gray bars) and RV-PA conduit (dark gray bars). mBTS, Modified Blalock-Taussig shunt; RV-PA, right ventricle to pulmonary artery. 


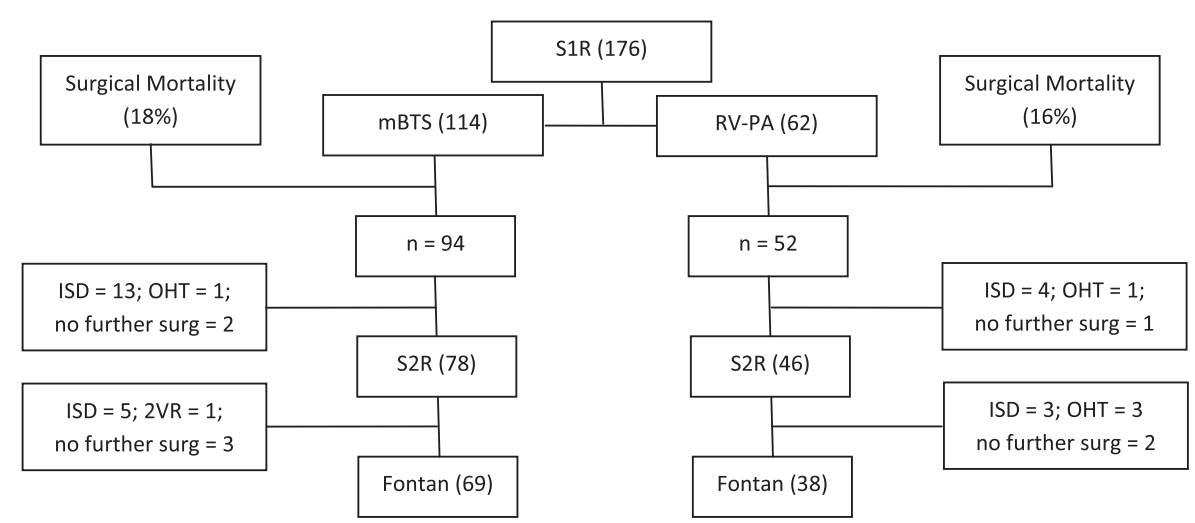

FIGURE 2. Outcomes of 176 patients who underwent S1R between January 1, 2002, and May 1, 2005. mBTS, Modified Blalock-Taussig shunt; RV-PA, right ventricle to pulmonary artery; ISD, interstage death; $O H T$, orthotopic heart transplant.

[14/35], $P=.009)$. Neoaortic valve regurgitation was not different between shunt types.

\section{Preoperative Cardiac Catheterization Data}

Eighty-one patients underwent preoperative cardiac catheterization (mBTS 77\% [53/69], RV-PA 74\% [28/38], $P=.9)$. Ten patients underwent magnetic resonance imaging evaluation, and the remaining patients were assessed only with echocardiography before Fontan. There was no difference in the mixed venous saturation between shunt types (mBTS 63\% [range 48\%-77\%]; RV-PA 60\% [range $45 \%-74 \%$ ], $P=.7$ ). Common atrial pressure (mBTS $7 \mathrm{~mm}$ $\mathrm{Hg}$ [range 3-13 mm Hg], RV-PA $8 \mathrm{~mm} \mathrm{Hg}$ [range 4-15 mm $\mathrm{Hg}], P=.04)$ and mean pulmonary artery pressure (mBTS $11 \mathrm{~mm} \mathrm{Hg}$ [range 6-24 mm Hg]; RV-PA $13 \mathrm{~mm} \mathrm{Hg}$ [range $8-24 \mathrm{~mm} \mathrm{Hg}], P=.03$ ) were significantly higher in patients with RV-PA conduits at the pre-Fontan catheterization. There was a trend toward higher single ventricle enddiastolic pressure (mBTS $6 \mathrm{~mm} \mathrm{Hg}$ [range 4-14 mm Hg]; RV-PA $8 \mathrm{~mm} \mathrm{Hg}$ [range $5-15 \mathrm{~mm} \mathrm{Hg}$ ], $P=.06$ ) in patients with RV-PA conduits.

\section{Fontan Operative Data and Morbidity}

The Fontan completion consisted of a fenestrated lateral tunnel Fontan or a fenestrated extracardiac conduit. There

TABLE 2. Preoperative patient characteristics

\begin{tabular}{lccc}
\hline & mBTS & RV-PA conduit & $\boldsymbol{P}$ value \\
\hline Age $(\mathrm{y})$ & $2.7(1.5-4.4)$ & $2.9(1.9-3.9)$ & .4 \\
Weight $(\mathrm{kg})$ & $13(9.2-17.6)$ & $13.5(11-17.6)$ & .3 \\
BSA $\left(\mathrm{m}^{2}\right)$ & $0.55(0.41-0.7)$ & $0.55(0.39-0.71)$ & .7 \\
Diuretic use & $26 / 69$ & $14 / 35$ & .5 \\
ACEI use & $45 / 69$ & $25 / 35$ & .3 \\
Digoxin use & $17 / 72$ & $16 / 37$ & .04 \\
Reflux medication use & $16 / 69$ & $7 / 35$ & .5 \\
NG or G-tube feeding & $8 / 69$ & $6 / 35$ & .3 \\
\hline
\end{tabular}

$m B T S$, Modified Blalock-Taussig shunt; $R V-P A$, right ventricle to pulmonary artery; $B S A$, body surface area; $A C E I$, angiotensin-converting enzyme inhibitor; $N G$, nasogastric; $G$, gastric. Data presented as median with range. $P$ values calculated using MannWhitney test. was no difference in total support time, aortic crossclamp time, or circulatory arrest time between shunt types (Table 3). The median total support time was 66 (range 52-195) minutes for the mBTS group and 64 (range 50-134) minutes for the RV-PA conduit group $(P=.19)$. Five patients underwent atrioventricular valvuloplasty at the time of Fontan (mBTS 2/69; RV-PA 3/38; $P=.5$ ).

There was no difference in the time to initial extubation, with the majority of the patients in both groups being extubated on arrival into the cardiac intensive care unit after Fontan completion. One patient in the RV-PA conduit group required reintubation for respiratory failure and eventual tracheostomy for subglottic stenosis. Two patients in the mBTS group required prolonged intubation ( 3 and 6 days, respectively) for low output state and eventual Fontan takedown related to Fontan baffle obstruction. Nine patients underwent cardiac catheterization during the same admission, after Fontan procedure (mBTS $7 / 69$, RV-PA $2 / 38 ; P=.6$ ); interventions including relief of arch obstruction $(\mathrm{n}=2)$ and stent placement in the left pulmonary artery $(\mathrm{n}=1)$ were performed. Other measures of morbidity included both hospital and cardiac intensive care unit lengths of stay. Neither measure was significantly different between groups, with a median length of hospital stay for both groups of 8 days (mBTS range 4-40 days, RV-PA 2-73 days; $P=.6$ ) and a median length of intensive care unit stay of 7 days (mBTS range $2-40$ days, RV-PA $1-73$ days; $P=.3$ ). Chest tubes were placed for pleural effusions in 21 of 69 patients in the mBTS group and 13 of 38 patients in the RV-PA conduit group $(P=.6$ ). Four patients (mBTS $3 / 69$, RV-PA $1 / 38$; $P=.9)$ required mediastinal incision and drainage.

There was an $18 \%$ rehospitalization rate within 30 days after initial discharge after Fontan completion (mBTS $15 \%$ [10/69]; RV-PA 16\% [6/38]; $P=.9$ ). Three patients with initial mBTS and 1 patient with RV-PA conduit had more than 1 readmission within 30 days of discharge, resulting in a higher total rehospitalization rate for the entire cohort than for 1 single shunt type. Median length of stay 
TABLE 3. Operative times at Fontan by shunt type

\begin{tabular}{lcl}
\hline & mBTS $(\mathbf{n}=\mathbf{6 2})$ & RV-PA conduit $(\mathbf{n}=\mathbf{3 1})$ \\
\hline $\begin{array}{l}\text { Total support } \\
\text { time (min) }\end{array}$ & $66(52-189)$ & $64(50-134) P=.07$ \\
$\begin{array}{l}\text { Aortic crossclamp } \\
\text { time (min) }\end{array}$ & $27(0-91)$ & $25(14-65) P=.12$ \\
DHCA (min) & $25(0-82)$ & $25(14-41) P=.08$ \\
\hline
\end{tabular}

$m B T S$, Modified Blalock-Taussig shunt; $R V-P A$, right ventricle to pulmonary artery conduit; $D H C A$, deep hypothermic circulatory arrest. Data presented as median with range. $P$ values calculated using Mann-Whitney test.

after readmission was not different between shunt types (mBTS median 7 days [range 2-36 days], RV-PA median 6.5 days [range $1-25$ days], $P=.4$ ). The reason for admission for all patients was pleural effusion, with 1 patient also having a superficial wound infection.

\section{Mortality and Follow-up}

There was $98 \%$ surgical survival for those patients undergoing Fontan completion. The 2 surgical deaths were in the mBTS group. One patient died of overwhelming gramnegative rod sepsis with uncontrollable hemorrhage. The second patient had a sudden cardiac arrest requiring extracorporeal membrane oxygenation cannulation with subsequent withdrawal of support secondary to embolic complications of heparin-induced thrombocytopenia.

The median duration of follow-up was 58 months (range 1-87 months). By Kaplan-Meier analysis, shunt type did not influence survival or freedom from transplant at 5 years (mBTS 70\%; 95\% confidence limit, 60-77, vs RV-PA $61 \%, 95 \%$ confidence limit, 47-72; $P=.55$; Figure 3 ). There have been no deaths or transplants in either group after Fontan completion and hospital discharge through the current follow-up.

Further analysis using Cox proportional hazards regression was performed to adjust for differences in baseline characteristics between the 2 groups (Table 4). Variables

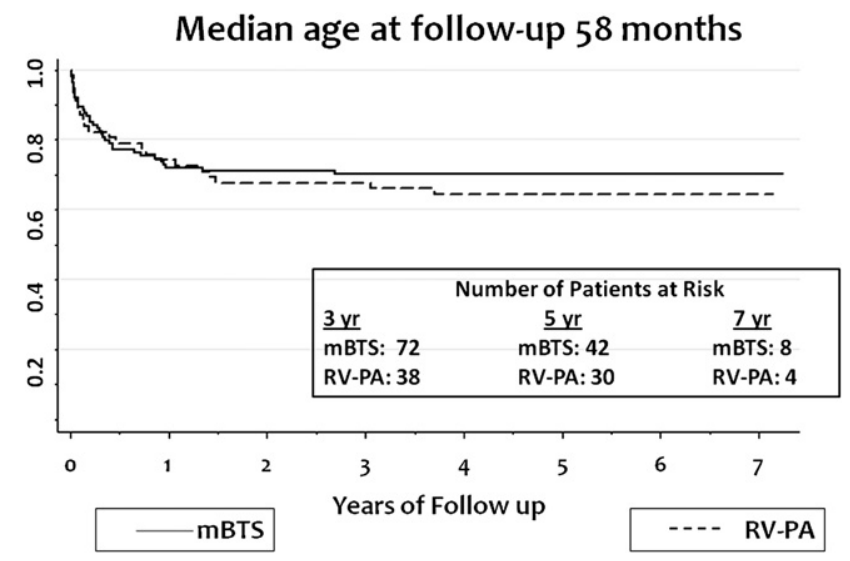

FIGURE 3. Kaplan-Meier freedom from death or transplant by shunt type. Start point is birth with follow-up to April 16, 2009.
TABLE 4. Cox proportional hazard regression models for death or transplant: Entire cohort

\begin{tabular}{lccc}
\hline \multicolumn{1}{c}{ Risk factor } & Hazard ratio & $\mathbf{9 5} \% \mathbf{C L}$ & $\boldsymbol{P}$ value \\
\hline Shunt type & 1 & $0.54-1.93$ & .96 \\
Single left ventricle & 0.30 & $0.07-1.32$ & .11 \\
Unbalanced AVC & 1.43 & $0.6-3.42$ & .42 \\
VSD with MS/MA & 0.79 & $0.32-1.98$ & .62 \\
Other & 0.42 & $0.054-5.26$ & .41 \\
Aortic atresia & 0.88 & $0.48-1.63$ & .69 \\
Associated cardiac & 2.5 & $1.19-5.26$ & .02 \\
$\quad$ risk factors & & & \\
Significant congenital & 4.5 & $1.96-10.21$ & $<.001$ \\
$\quad$ anomalies & & & \\
Defined genetic syndrome & 0.29 & $0.04-2.17$ & .23 \\
Birth weight $<2.5 \mathrm{~kg}$ & 3.19 & $1.8-5.8$ & $<.0001$ \\
Shock at birth & 1.72 & $0.76-3.92$ & .19 \\
\hline
\end{tabular}

$A V C$, Atrioventricular canal; $C L$, confidence limit; $V S D$, ventricular septal defect; $M S$, mitral stenosis; $M A$, mitral atresia. Shunt type is RV-PA compared with mBTS baseline. Other $=$ unbalanced AVC with truncus arteriosus and interrupted aortic arch, superior ventricles with $A V C$ and transposition of the great arteries $\{S, D, L\}$ with mitral stenosis. Additional cardiac risk factors were considered to be an intact atrial septum requiring emergency intervention, moderate to severe AVVR, and severe ventricular dysfunction at birth. Noncardiac anomalies included scimitar syndrome, gastroschisis, duodenal atresia, and tracheoesophageal fistula.

added into the model included cardiac diagnosis, additional cardiac risk factors, significant noncardiac anomalies, and aortic atresia (Table 1). By adjusting for these differences in baseline characteristics, there was no significant association between shunt type and death or transplant (hazard ratio $1.0 ; 95 \%$ confidence interval, 0.54-1.93). By restricting the model to only those patients with HLHS, there was still no significant association between shunt type and outcome (hazard ratio $1.17 ; 95 \%$ confidence interval, 0.54-2.44).

\section{DISCUSSION}

This most recent review of this contemporary cohort of patients demonstrates several significant findings. There was significantly more qualitative ventricular dysfunction and AVVR in those patients who underwent RV-PA conduit as part of the S1R. These patients also had significantly higher common atrial and mean pulmonary artery pressures at the time of pre-Fontan catheterization. However, despite these significant differences, the ability to tolerate Fontan completion was not different between shunt groups. In addition, freedom from death or transplant was not different between groups, with a median follow-up time of approximately 5 years. Multivariate analysis did not demonstrate a protective effect of the anatomic subtype of single left ventricle or of one shunt type over the other. As previously reported, the presence of additional cardiac risk factors and noncardiac congenital anomalies was highly predictive of worse outcome. ${ }^{1}$ The pattern of interstage death and transplantation at this point in follow-up seems to demonstrate a trend toward early attrition in the group with mBTS and late attrition in the RV-PA conduit group, which may be 
a result of longer-term effects of the ventricular dysfunction noted in the RV-PA group.

Multiple factors have resulted in improved survival among infants born with functional single-ventricle lesions. These factors include surgical modifications, improved surgical technique, modifications to preoperative and postoperative care, and a better understanding of the cardiac physiology of a patients with single-ventricle lesions undergoing surgical reconstruction. ${ }^{6-11}$ The most recent modification is the use of an RV-PA conduit. This technique was first described by Norwood and colleagues ${ }^{12}$ in the early 1980s but was abandoned in favor of a systemic to pulmonary shunt. In recent years, multiple centers have reported improved surgical survival with the use of this conduit when compared with historical controls through the first interstage period. ${ }^{13,14}$ Several large centers have published contemporary series that have not demonstrated the benefit of either shunt strategy through S1R and S2R..$^{1-3}$

Few reports are available detailing intermediate and longer-term outcomes after the use of RV-PA conduit at S1R through Fontan completion. Januszewska and colleagues ${ }^{4}$ reported no difference in mortality, ventricular function, arrhythmia, or length of stay in a consecutive series of 50 patients with HLHS at the time of Fontan completion. In addition, a more recent report of a series of 80 patients who contemporaneously underwent S1R with either mBTS or RV-PA conduit did not demonstrate a statistically significant difference in outcome between shunt types. ${ }^{5} \mathrm{We}$ report our single-institution experience with Fontan completion and overall survival in a cohort of patients who underwent S1R with either an mBTS or RV-PA conduit in the same era with a median follow-up of 4 years.

Kaplan-Meier 5-year survival for the entire series cohort is $68 \%$ (95\% confidence limit, 58-75). A total of 107 patients underwent Fontan completion with $98 \%$ early survival. As demonstrated by Kaplan-Meier analysis, shunt type did not influence survival or freedom from transplant at 5 years (Figure 3). Although a larger number of patients with RV-PA conduit underwent transplantation, this was not statistically significant. All patients received transplants as a result of severe ventricular dysfunction. Survival in our cohort is similar to that reported in both historically controlled and contemporary series of Fontan completion using both shunt types. ${ }^{5,15}$ This early survival is also similar to that previously reported in large, single institutional reviews of Fontan outcomes after an initial Norwood procedure with mBTS. $^{16,17}$

Qualitative ventricular dysfunction was worse in those patients palliated initially with RV-PA conduit in comparison with mBTS with nearly twice as many patients in the RV-PA conduit group having an assessment of moderate to severe ventricular dysfunction at the time of preoperative Fontan evaluation. This association remained as strong at the time of Fontan completion as it was at the time of S2R. In the interim period, 3 patients with an RV-PA conduit and significant ventricular dysfunction underwent transplantation. It remains unclear whether the ventriculotomy of the RV-PA conduit is the cause of the impaired systolic ventricular performance over time. These findings are in contrast with those published by Boston Children's Hospital, which reported a trend toward improved outcome with the use of the RVPA conduit. ${ }^{5}$ In addition, although AVVR was significantly worse at the time of Fontan completion in those patients with a previous RV-PA conduit, it did not result in an inability to undergo successful Fontan surgery. Marginally higher but statistically significant, mean pulmonary artery pressure and filling pressure at the time of the pre-Fontan cardiac catheterization in those patients with a previous RV-PA conduit did not limit Fontan suitability or successful completion.

Other preoperative patient characteristics, including need for supplemental feeding, need for ancillary therapies, medication use, age, and size at time of Fontan completion did not differ between the 2 shunt types. Intraoperative times were not different between groups, and the recovery from surgery documented as time to extubation and length of stay was not different between initial shunt types. Incidence of pleural effusions, length of drainage, and need for rehospitalization for pleural effusions were similar between groups. Pleural effusions did not seem to be related to pulmonary artery architecture because only 1 patient required an interventional intervention to relieve stenosis in the postoperative Fontan period. There was no statistical association between the presence of pleural effusions and the preoperative pulmonary artery pressure or ventricular end-diastolic pressure.

\section{Study Limitations}

This study is limited by its retrospective nature and the biases inherent in this type of study design. This is a nonrandomized patient population, with possible uneven distribution of unrecognized preoperative risk factors between shunt groups. As previously stated, all patients with single left ventricles were initially palliated with $\mathrm{mBTS}$, and there was a preference for the use of an RV-PA conduit in those patients with aortic atresia. Although this was a contemporary rather than historical comparison of shunt types, there was uneven distribution of shunt types over time. Although few patients were lost to follow-up, patients did not receive the same preoperative evaluation, and as such some data analysis may be biased. Echocardiographic data are what were documented in the most recent report before Fontan procedure. Images were not reviewed at the time of collection, which introduces the bias of multiple readers and data interpretation. It is a recent study group, and important late outcomes, such as arrhythmia, sudden death, and ventricular dysfunction, might not have yet occurred. This study might not be powered to evaluate small differences between comparison groups. 


\section{CONCLUSIONS}

The data of this contemporary cohort of patients do not support the use of one shunt type over the other at the time of the S1R as it relates to ability to proceed to Fontan completion. Overall survival between shunt types remains similar through Fontan completion; however, timing of death and morbidity are different. There may be a survival advantage early in the palliation period for patients with RV-PA conduits, but this seems to diminish over time with continued attrition with death and transplantation after S2R. In addition, ventricular dysfunction and AVVR continue to be significant in those patients with RV-PA conduits at the time of Fontan completion. This may contribute significantly to longer-term outcomes. As such, this limited study group, with the inherent biases to this type of retrospective study and the relatively short follow-up time period, emphasizes the need for longitudinal follow-up in a randomized, controlled trial, such as the Single Ventricle Reconstruction trial, which is ongoing at the current time. ${ }^{18}$

\section{References}

1. Tabbutt S, Dominguez TE, Ravishankar C, Marino BS, Gruber PJ, Wernovsky G, et al. Outcomes after the stage I reconstruction comparing the right ventricular to pulmonary artery conduit with the modified Blalock Taussig shunt. Ann Thorac Surg. 2005;80:1582-91.

2. Ballweg JA, Dominguez TE, Ravishankar C, Kreutzer J, Marino BS, Bird GL, et al. A contemporary comparison of the effect of shunt type in hypoplastic left heart syndrome on the hemodynamics and outcome at stage 2 reconstruction. J Thorac Cardiovasc Surg. 2007;134:297-303.

3. Lai L, Laussen PC, Cua CL, Wessel DL, Costello JM, del Nido PJ, et al. Outcomes after bidirectional Glenn operation: Blalock-Taussig shunt versus right ventricle-to-pulmonary artery conduit. Ann Thorac Surg. 2007;83:1768-73.

4. Januszewska K, Stebel A, Malec E. Consequences of right ventricleto-pulmonary artery shunt at the first stage for the Fontan operation. Ann Thorac Surg. 2007;84:1611-7.

5. Scheurer MA, Salvin JW, Vida VL, Fynn-Thompson F, Bacha EA, Pigula FA, et al. Survival and clinical course at Fontan after stage one palliation with either a modified Blalock-Taussig shunt or a right ventricle to pulmonary artery conduit. $J$ Am Coll Cardiol. 2008;52:52-9.

6. Bove EL, Lloyd TR. Staged reconstruction for hypoplastic left heart syndrome. Contemporary results. Ann Surg. 1996;224:387-95.

7. Bove EL. Current status of staged reconstruction for hypoplastic left heart syndrome. Pediatr Cardiol. 1998;19:308-15.

8. Mahle WT, Spray TL, Wernovsky G, Gaynor JW, Clark BJ. Survival after reconstructive surgery for hypoplastic left heart syndrome: a 15 year experience from a single institution. Circulation. 2000;102(suppl 3):III36-41.

9. Malec E, Januszewska K, Kolz J, Pajak J. Factors influencing early outcome of Norwood procedure for hypoplastic left heart syndrome. Eur J Cardiothorac Surg. 2000;18:202-6

10. Azakie A, Sl Merklinger, McCrindle BW, Van Arsdell GS, Lee KJ, Benson LN, et al. Evolving strategies and improving outcomes of the modified Norwood procedure: a 10 year single institution experience. Ann Thorac Surg. 2001;72:1349-53.

11. Tweddell JS, Hoffman GM, Mussatto KA, Fedderly RT, Berger S, Jaquiss RD, et al. Improved survival of patients undergoing palliation of hypoplastic left heart syndrome: lessons learned from 115 consecutive patients. Circulation. 2002; 106(suppl I):I82-9.

12. Norwood WI, Lang P, Castaneda AR, Campbell DN. Experience with operations for hypoplastic left heart syndrome. J Thorac Cardiovasc Surg. 1981;82:511-9.

13. Sano S, Ishino K, Kawada M, Arai S, Kasahara S, Asai T, et al. Right ventriclepulmonary artery shunt in first-stage palliation of hypoplastic left heart syndrome. J Thorac Cardiovasc Surg. 2003;126:504-10.

14. Sano S, Ishino K, Kado H, Shiokawa Y, Sakamoto K, Yokota M, et al. Outcome of right ventricle to pulmonary artery shunt in first stage palliation of hypoplastic left heart syndrome: a multi-institutional study. Ann Thorac Surg. 2004;78: 1951-8.
15. Sano S, Ishino K, Kawada M, Yoshizumi K, Takeuchi M, Ohtsuki S. Experience over five years using a shunt placed between the right ventricle and the pulmonary arteries during initial reconstruction of hypoplasia of the left heart. Cardiol Young. 2004; 14(Suppl 3):90-5.

16. Mitchell ME, Ittenbach RF, Gaynor JW, Wernovsky G, Nicolson SC, Spray TL. Intermediate outcomes after the Fontan procedure in the current era. $J$ Thorac Cardiovasc Surg. 2006;131:172-80.

17. Hirsch JC, Goldberg C, Bove EL, Salehian S, Lee T, Ohye RG, et al. Fontan operation in the current era. Ann Surg. 2008;248:402-10.

18. Ohye RG, Gaynor JW, Ghanayem NS, Goldberg CS, Laussen PC, Frommelt PC, et al. Design and rationale of a randomized trial comparing the Blalock-Taussig and right ventricle-pulmonary artery shunts in the Norwood procedure. $J$ Thorac Cardiovasc Surg. 2008;136:968-75.

\section{Discussion}

Dr Christian Pizarro (Wilmington, Del). I congratulate Dr Ballweg and colleagues on a timely study comparing the outcomes of patients who underwent Fontan completion after receiving alternative sources of pulmonary blood flow at the time of their Norwood procedure. While the pediatric cardiac surgical community awaits the results of the Single Ventricle Reconstruction trial, these data provide important information about the impact of each shunt on the candidacy of these patients to undergo a Fontan procedure and their operative results and midterm outcome.

The authors conclude that there is no evidence to support the use of one shunt over the other; nevertheless, each group presented a different pattern on the timing of death and morbidity. Although there were more interstage deaths in the mBTS group, when using a composite end point of death or transplantation, this difference is neutralized by the fact that 3 patients in the RV-PA group underwent transplantation after their second stage.

My first question, based on these different patterns of attrition, is did you identify any specific subgroup of patients who would benefit the most with one specific type of shunt?

Dr Ballweg. No, we did not. We haven't looked back at the data and teased out if patients with aortic atresia, those with mitral and aortic atresia, or the group that some people have reported as higher risk (ie, mitral stenosis and aortic atresia) would benefit from one shunt type or the other at this point.

Dr Pizarro. Thank you, considering the difficulty related to the objective evaluation of right ventricular function, the data presented show a substantially higher incidence of moderate to severe right ventricular systolic dysfunction in the RV-PA shunt group, a finding that has not consistently been reported in other series, particularly the one that you have mentioned. A review of our own experience, comparing the outcome of shunt types 3 years after the Fontan, presented at the Society of Thoracic Surgeons last year, did not detect any differences in systolic function; however, the incidence and duration of pleural effusions showed a tight correlation with a history of balloon intervention for recoarctation.

Have you had the opportunity to assess and compare the diastolic properties of these ventricles, and have you observed a similar phenomenon?

Dr Ballweg. I'll answer the second part first. In regard to the data you presented with coarctation and pleural effusions, there were interventions on arch obstruction in these patients, but it was mainly after Fontan. We have had some patients who also had pre-Fontan arch obstruction that was relieved, and those patients did not have increased duration or incidence of pleural effusion. Those data are 
not discussed in this article but will be presented in June at the Western Thoracic Surgical Association meeting.

In regard to your first question, I'm not sure we have a good method of evaluating diastolic dysfunction, quite honestly. I think the data showing that the end-diastolic pressure and left atrial pressure are increased are certainly markers that the diastolic function of the ventricles may be worse for those with an RV-PA conduit at stage 1 .

Dr Pizarro. As longer follow-up is available, the issues of late failure and need for transplantation become matters of special interest. The Michigan group and others have shown that the outcome of patients with tricuspid valve regurgitation after the Norwood is closely related to the degree of ventricular dysfunction; therefore, attempts to correct the tricuspid regurgitation should be undertaken early. Given the fact that $40 \%$ of the patients in the RV-PA shunt group had important tricuspid regurgitation, could you tell us how these patients were managed? Did they undergo any attempt at tricuspid valve repair before or at the time of Fontan, or have you developed a protocol to address this important issue?

Dr Ballweg. In our previous discussion and publication at stage 2, we had 10 patients who had undergone tricuspid valvuloplasty, 4 were in the mBTS group and 6 were in the RV-PA conduit group.

Unfortunately, I don't have the specific data on how many tricuspid valvuloplasties were done at the time of the Fontan. I can tell you of only one that I can recall. And in discussing this with other people who are here at the meeting with me, it's rare for us to intervene on tricuspid regurgitation at the time of Fontan. It has to be extremely severe; however, you want to define that, and certainly it's not the norm. So although we had a large percentage of patients with tricuspid regurgitation by echo definition of multiple reviewers, we did not intervene.

As far as management, I think there is a certain camp that tends to afterload reduce patients with AVVR more aggressively than others. We do not have a standard protocol, but I think people are aware that oftentimes afterload reduction may be helpful.

Dr Pizarro. We learned from your previous report on this cohort of patients that those in the RV-PA group were younger and had lower arterial saturations at stage 2 . Did you observe any difference in the degree of systemic to pulmonary collaterals present in these patients before Fontan completion, and were there any differences in the interventions undertaken?

Dr Ballweg. That wasn't the objective of our study, and we didn't look for it. I don't have those data to present today.

Dr Emile Bacha (Boston, Mass). I wonder if you're not measuring 2 different groups of patients. I may have missed it in the presentation. You probably know that tomorrow we're presenting out of Boston a similar series of patients with a bit longer follow-up, and we found the exact opposite in terms of tricuspid regurgitation rates.
Now, you showed that approximately two thirds of your patients had HLHS; the rest had single left ventricles (LVs) and so forth. Those are patients in whom we would tend to perform mBTS. Those are patients who tend to do much better in terms of AVVR and ventricular function. So by comparing these 2 groups, I'm assuming that you have more single LVs in the mBTS group. I may be wrong, but that's my assumption, and I wonder if you would repeat this analysis with only patients with HLHS.

Dr Ballweg. There were 9 patients with single LVs, and they are all in the mBTS group. Certainly we could exclude them and redo the analysis just with HLHS and variants. The variants were the more common things we see in such an unbalanced canal.

Dr Bacha. I would encourage you to do that because it would be interesting to see what you find.

Dr Shunji Sano (Okayama City, Japan). The LV dysfunction and tricuspid regurgitation are concerns for the RV-PA shunt. But I think the LV dysfunction and tricuspid regurgitation can be avoided mainly in 2 ways. One is the LV incision. We changed the technique from excising the LV, the muscle, from the scissors of knife to punch out the muscle, because it's more accurate and the damage to the LV is minimal, rather than the cutting knife with scissors.

The second one is that the volume overload is also avoided by putting a clip to the graft to control the saturation. When the operation is finished, we keep the saturation between $80 \%$ and $85 \%$ with a clip, mostly to a 5-mm graft, because our patient population is smaller than yours. So the volume overload could be controlled. Then afterward, when the patients grow up, the cardiologists do balloon dilate and remove the clip so that we can wait a little more because the average body weight at Fontan is only $10 \mathrm{~kg}$. So there are 2 ways we can somehow control or manage or minimize the LV dysfunction and tricuspid regurgitation.

What is your technique of LV incision? What size graft do you use in an RV-PA shunt? When you finish the RV-PA shunt, how much is the saturation, and can you try to control the saturation to control the volume overload?

Dr Ballweg. We do not use the punch technique. So if you have seen a change in ventricular dysfunction and AVVR, it may be a better option. We do not do anything to control the pulmonary blood flow through the Sano conduit at the time the operation is completed. I believe the majority of our conduits are $5 \mathrm{~mm}$ as well. Our saturations are generally in the mid to high 80 s when patients leave the operating room.

Dr Frank Hanley (Stanford, Calif). Was there any correlation between the reduced right ventricular function by ejection fraction determined by echocardiography and the right ventricular end-diastolic pressure at the pre-Fontan catheterization?

Dr Ballweg. No, there was not. 